Abb. 1 Wer komplementäre Arzneien anwendet, sollte eines immer bedenken: Sie können mit schulmedizinischen Wirkstoffen interagieren! Foto: (C) fotolia/zest_marina

\title{
Mit Bedacht kombinieren
}

\section{Zwischen Schul- und Komplementärmedizin kann es zu WECHSELWIRKUNGEN kommen. Deshalb sollte am Anfang jeder Therapie eine gründliche Anamnese stehen.}

\author{
Peter Holzhauer
}

WECHSELWIRKUNGEN zwischen Arzneimitteln können erwünscht sein, um durch die Kombination mehrerer Wirkstoffe das jeweilige Behandlungsziel optimaler zu erreichen. Das trifft zum Beispiel auf zahlreiche Kombinationen von blutdrucksenkenden Medikamenten zu. Die synergistische Wirkung der Einzelsubstanzen führt hier zu einer effektiveren Senkung des Blutdrucks. Doch auch unerwünschte Arzneimittelinteraktionen kommen häufig vor. Bei der heute üblichen Polypharmazie, also der paral- lelen Einnahme verschiedenster Wirkstoffe gerade bei älteren und polymorbiden Patienten, sind unerwünschte Wechselwirkungen unbedingt $\mathrm{zu}$ beachten und durch gute kompetente Verordnung zu vermeiden [5].

\section{Welche Wechselwirkungen gibt es?}

Wechselwirkungen können verschiedener Natur sein. Sie entstehen durch die
Passage und den Stoffwechsel im Körper oder treten beim gemeinsamen Verabreichen verschiedener Medikamente auf.

Pharmakokinetische Wechselwirkungen

Das Prinzip der pharmakokinetischen Wechselwirkungen betrachtet die Veränderung eines Wirkstoffs während der Passage und des Stoffwechsels im Körper. Arzneimittel unterliegen pharmakokinetischen Veränderungen vom Beginn der Einnahme bis zur Ausscheidung aus dem 
Körper. Einflussgrößen und Variablen in diesem System sind Resorption, Verteilung und Metabolisierung, zum Beispiel durch das Cytochrom-P450-Enzymsystem (siehe Kasten). Außerdem spielt die Elimination durch Lunge, Leber und Niere sowie durch Proteine eine wichtige Rolle. Zu Letzteren gehört unter anderem das P-Glykoprotein. Es steht für eine Familie membranständiger Transportproteine, die Medikamente aktiv aus der Zelle entfernen können.

Pharmakokinetische Wechselwirkungen kommen sehr häufig vor. Besonders relevant sind die zahlreichen Interaktionen, die durch den Einfluss von Medikamenten auf die Aktivität von Enzymen der Cytochrom-P450-Familie bedingt sind. Bekannte Beispiele sind hier die Interaktionen von Grapefruitsaft mit verschiedenen Zytostatika über das Cytochrom-P450-System [5].

\section{KURZ GEFASST}

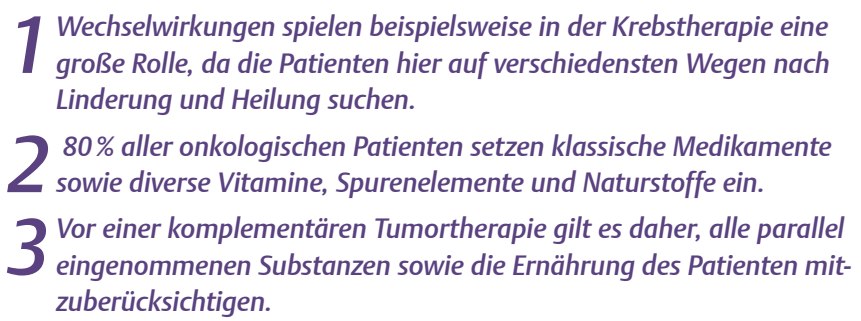

\section{Pharmakodynamische Wechsel- wirkungen}

Pharmakodynamische Wechselwirkungen können auftreten, wenn zwei unterschiedliche, gleichzeitig verabreichte Medikamente miteinander in Konkurrenz treten, zum Beispiel an der Bindungsstelle eines Rezeptors oder einer anderen Zielstruktur. Wenn zwei Arzneistoffe mit gleichem Wirkprofil unerwünschte, sich verstärkende Effekte aus-

\section{Das Cytochrom-P450-System}

Rund 60 Enzyme sind heute bekannt, die die sogenannte Cytochrom-P450-Familie bilden. Sie sind für den Abbau körpereigener und körperfremder Substanzen zuständig und kommen vor allem in der Leber, aber auch in Darm, Niere und Lunge vor. Sie ermöglichen die Hydroxylierung von Verbindungen und machen damit hydrophobe Substanzen wie Medikamente wasserlöslich. So können diese über Niere und Galle ausgeschieden werden.

Das mengenmäßig wichtigste Enzym der Cytochrom-P450-Familie in der Leber ist das CYP3A4. Etwa die Hälfte aller Arzneimittelwirkstoffe wird über dieses Enzym verstoffwechselt, was es besonders anfällig für Wechselwirkungen macht. Dabei können die Wirkstoffe induzierend oder inhibierend auf die Enzymaktivität wirken. Bei der Induktion erhöht sich die Konzentration von CYP3A4. Der Arzneimittelwirkstoff kann schneller abgebaut werden, und die notwendige Konzentration im Blut reicht eventuell nicht mehr aus. Bei der Inhibition ist genau das Gegenteil der Fall: Die Enzymaktivität wird gehemmt, und der Medikamentenwirkstoff reichert sich an. Dadurch kann eine toxische Wirkung auftreten.

Ein Beispiel für einen inhibierenden Wirkstoff ist Grapefruitsaft: $\mathrm{Er}$ führt zu einer irreversiblen Hemmung von CYP3A4 in der Darmwand (zu beachtende Zeit der Hemmung liegt zwischen 4 und 24 Stunden). Bei oraler Einnahme bestimmter Medikamente, wie Zytostatika, erhöht sich deren Bioverfügbarkeit, da die Regulierung durch das CYP3A4 entfällt.

Weitere wichtige Enzyme der Cytochrom-P450-Familie sind CYP2D6, CYP2C9, CYP2C19 sowie CYP1A2. üben, ist ebenfalls von pharmakodynamischen Interaktionen die Rede. Dies ist auch der Fall, wenn zwei Medikamente mit unterschiedlichem Wirkprofil, beispielsweise ein Abführmittel und ein Diuretikum, einen unerwünschten Effekt wie eine Hypokaliämie auslösen. Solche Wechselwirkungen können in der Folge durchaus klinische Relevanz haben [5].

\section{Pharmazeutische Wechsel- wirkungen}

Wenn zwei oder mehrere Medikamente gemeinsam in einer Infusionslösung verabreicht werden und diese Medikamente inkompatibel sind, dann liegt eine pharmazeutische Arzneimittelinteraktion vor. Diese Art der Wechselwirkungen sind sehr bedeutend und können zum Beispiel bei einer Mischinfusion mit Natriumselenit (Selen) und Vitamin C auftreten.

\section{Achtung: Um pharmazeutische Wechselwirkungen zu umgehen, Mischinfusionen immer vermei- den! Besser: Die entsprechenden Wirkstoffe einzeln und getrennt infundieren [5].}

Beachten Sie auch: Wenn zwei oder mehr Arzneimittel in einer Infusion kombiniert werden, entsteht dadurch ein neues. Die Hersteller der einzelnen Medikamente sind damit dann für eventuelle Schäden nicht mehr haftbar.

\section{Wechselwirkungen in der Krebstherapie}

Viele Anwender der klassischen medikamentösen Tumortherapie haben Bedenken bezüglich des gleichzeitigen Einsat- 


\section{Glossar}

Prodrug: Ein Prodrug ist pharmakologisch ein Vorläufer des eigentlichen Wirkstoffs. Er besitzt wenig oder keine aktive Wirkung und wird erst durch die Verstoffwechslung im Körper zur aktiven Substanz.

Topoisomerase: Topoisomerasen sind Enzyme, die die doppelläufigen DNA-Stränge vorübergehend spalten können und damit wichtige räumliche Voraussetzungen für das Ablesen der DNA (Transskription) schaffen.

Tubulin-Polymerisation: Tubuline sind Bausteine der Mikrotubuli und damit verantwortlich für die mechanische Stabilität der Zelle. Durch Polymerisation, also durch eine Kettenreaktion, entstehen aus einzel- nen molekularen Komponenten, in diesem Fall verschiedene Tubuline, röhrenförmige Mikrotubuli. Diese sind wesentlich am Prozess der Zellteilung beteiligt.

Monoklonale Antikörper: Monoklonale Antikörper werden seit Ende der 1970erJahre biosynthetisch in größerem Umfang hergestellt. Dabei setzt man das ypsilonförmige Immunglobulin G als Arzneistoff ein. Heute ist es möglich, monoklonale Antikörper gegen jede beliebige Zielstruktur, beispielsweise auf Zelloberflächen, herzustellen. Einsatz finden diese modernsten, gezielt wirkenden Medikamente vor allem in der Krebstherapie, aber auch in der Rheumatologie und Neurologie.
Lumisterol, Tachysterol: Lumisterol und Tachysterol sind steroide Naturstoffe, die im Vitamin-D-Stoffwechsel vorkommen.

Pregnan-X-Rezeptor: Der Pregnan-X-Rezeptor ist ein zellkernständiger Rezeptor, der wesentlich an der Aufspürung und Entgiftung von toxischen, körperfremden Substanzen beteiligt ist.

Glutathiontransferasen: Glutathiontransferasen sind leberständige Entgiftungsenzyme, die die Konjugation von körperfremden organischen Stoffen mit Glutathion fördern und damit wesentlich zur Entgiftung des Körpers beitragen. zes von Antioxidanzien und klassischen Zytostatika. Dabei sind die antineoplastischen Wirkmechanismen heute sehr gut untersucht. Bei den meisten Wirkstoffen spielt die Generation von Sauerstoffradikalen zwar eine Rolle, sie ist aber nur ein Begleitphänomen und nicht der wesentliche Wirkeffekt. Die genauen Wirkungsmechanismen sind nachstehend bei den einzelnen Zytostatika unter zytotoxischen Effekten beschrieben [6].

Alkylanzien

- Cyclophosphamid

- Bendamustin

- Platinsalze

Zytotoxische Effekte:

- DNA-Veränderungen

- Vernetzung von DNA-Strängen

- abnorme Basenpaarung

- Crosslinks

- Strangbrüche

\section{Interaktionspotenzial:}

- Cyclophosphamid und Johanniskraut führen zu einer CYP3A4-Induktion und somit zu einer Wirkungsverstärkung.

- Prodrug Cyclophosphamid und Grapefruitsaft führen zu einer CYP3A4-Inhibition und somit zu einer
Wirkungsabschwächung, da das Prodrug nicht ausreichend in die aktive Substanz überführt wird.

- Prodrug Bendamustin und CYP1A2Inhibitoren wie Cimetidin, Acyclovir, Ciprofloxacin oder Cisplatin führen zu einer Wirkungsabschwächung durch Acetylcystein (ACC).

\section{Antimetabolite}

- Methotrexat

- 5-Fluorouracil (5-FU)

- Gemcitabin

- Pemetrexed

Zytotoxische Effekte:

- Störung der DNA-Synthese

- Hemmung von Enzymen

- Integration „falscher“ DNA-Bausteine

\section{Interaktionspotenzial:}

- Methotrexat führt zu Abschwächung durch Glutamin und Folsäure.

- 5-FU führt zu Abschwächung durch Vitamin A; schwere Nebenwirkungen bei der Einnahme des Virusstatikums Brivudin (Zostex).

Anthrazykline

- Doxorubicin

- Epirubicin

- Idarubicin
Zytotoxische Effekte:

- Störung der DNA-Synthese

- Hauptmechanismus ist die Hemmung der Topoisomerase II.

- Erzeugung konsekutiver DNA-Strangbrüche

Interaktionspotenzial:

- Doxorubicin führt zu Abschwächung durch die Vitamine A, C und E sowie durch Glutamin.

- Störung der Biosynthese von L-Carnitin; in der Folge kann ein L-CarnitinMangel entstehen

Mitosehemmstoffe

- Vincaalkaloide

- Vinorelbin, Vindesin

- Taxane

Zytotoxische Effekte:

- Mitose-Gifte/Spindelgifte

- Hemmung der Tubulin-Polymerisation

\section{Interaktionspotenzial:}

- Taxane greifen negativ in den Vitamin-D-Stoffwechsel ein und können zu Vitamin-D-Mangel mit erhöhter Toxizität der Chemotherapie führen.

- Vinorelbin wird über CYP3A4 metabolisiert. 
Antibiotika

- Bleomycin

- Mitomycin

Zytotoxische Effekte:

- Alkylierung beziehungsweise Quervernetzung von DNA-Strängen

- Bleomycin führt zu Freisetzung eines Wasserstoffradikals, also einer oxidativen Reaktion, die möglicherweise durch therapeutisch verabreichte Antioxidanzien beeinträchtigt würde.

- Mitomycin führt zu DNA-Crosslinks unter vorheriger Reduktion; diese Effekte werden durch oxidative Intermediate ausgeübt, das heißt, hier sind oxidativ wirksame Effekte kausal am Wirkungsmechanismus beteiligt. In diesem Fall könnte eine begleitende Behandlung mit Antioxidanzien kontraproduktiv sein.

Achtung: Wechselwirkungen oder Wirkungsabschwächungen von Antibiotika mit klassischen Antioxidanzien sind potenziell möglich!
Topoisomerasehemmer

- Topotecan

- Irinotecan

- Etoposid

Zytotoxische Effekte:

Die Zielstruktur kann durch Hemmung der Topoisomerase I und II beeinträchtigt werden.

Achtung: Glutathion führt zu Wirkungsabschwächung von Etoposid und Etoposid-Metaboliten [18].

Interaktionspotenzial:

- Inrinotecan wird über CYP3A4 metabolisiert; Achtung: Johanniskraut führt zu CYP3A4-Induktion und somit zu Wirkungsabschwächung.

- Etoposid wird über CYP3A4 metabolisiert; Achtung: Grapefruitsaft führt zu CYP3A4-Inhibition und Johanniskraut zu CYP3A4-Induktion.

Achtung: Johanniskraut und Grapefruitsaft nicht gleichzeitig anwenden!

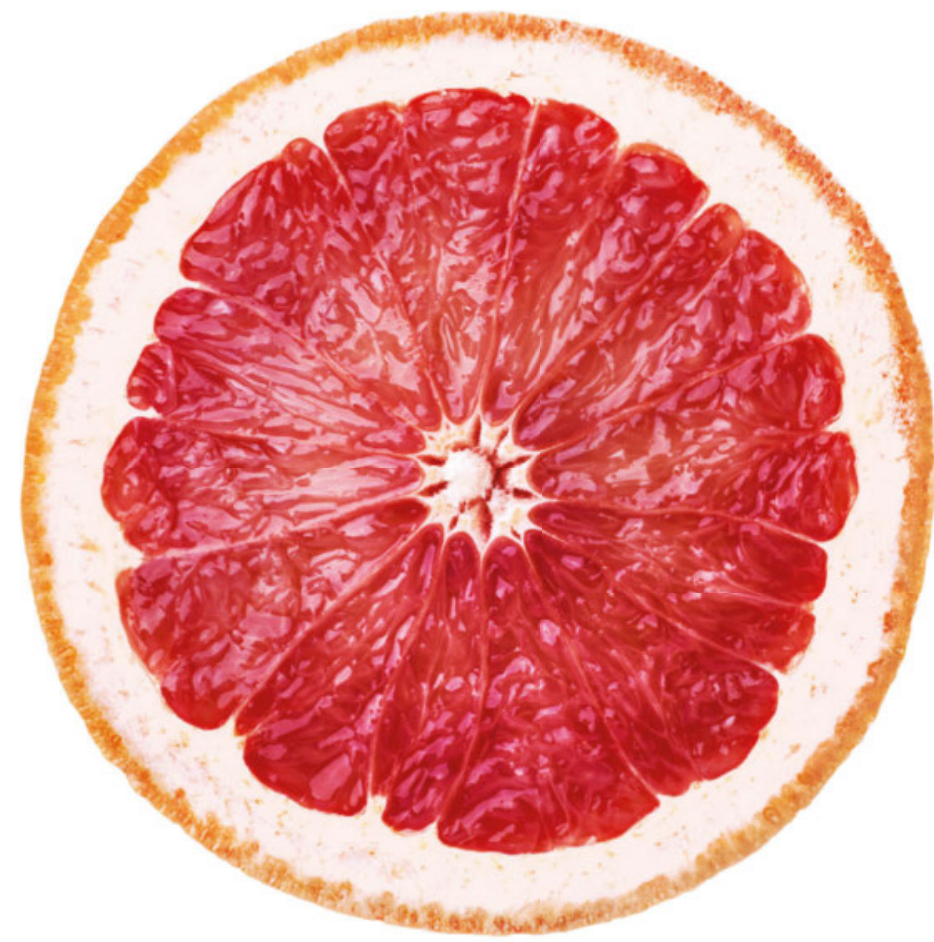

Abb. 2 Grapefruitsaft hemmt CYP3A4. Werden bestimmte Medikamente, wie Zytostatika, gleichzeitig eingenommen, kann deren Bioverfügbarkeit erhöht sein, da die Regulierung durch das Enzym entfällt. Foto: ㄷ fotolia/Yury Zap
Monoklonale Antikörper (Auswahl)

- Cetuximab

- Rituximab

- Trastuzumab

- Bevacizumab

- Pembrolizumab

- Nivolumab

Metabolisierung, Interaktionspotenzial, Synergien:

Monoklonale Antikörper sind heute therapeutisch sehr weit verbreitet. Sie werden nicht über das Cytochrom-P450-System verstoffwechselt und zeigen keine Interaktionen mit membranständigen Transportproteinen. Deshalb ist das Interaktionspotenzial sehr gering.

Merke: Bei defizitärer Versorgung mit Vitamin D und Selen kann die antikörpervermittelte Zytotoxizität eingeschränkt sein und eine Wirkungsabschwächung entstehen.

\section{Naturheilkundliche und kom- plementäre Begleittherapien}

Begleitend zur klassischen medikamentösen Tumortherapie nutzen viele Patienten naturheilkundliche und komplementärmedizinische Behandlungen. Gerade die Supplementierung von Mikronährstoffen und Naturstoffen findet häufig Anwendung.

Selen

Zufuhr in Form von Natriumselenit

Indikationen in der Komplementärmedizin:

- Supplementierung bei Selenmangel

- interventionelle, begleitende Gabe zur Strahlentherapie. Selen als Natriumselenit bewirkt hier über die sehr komplexe Einbindung in DNA-Reparatursysteme den selektiven Schutz gesunder, genetisch stabiler Zellen.

- interventionelle, begleitende Gabe zur Chemotherapie. Abhängig von der gewählten Dosierung und der Verwendung von anorganischem Selen als Natriumselenit werden auch hier 
DNA-Reparatursysteme unterstützt, und auf diesem Weg wird eine selektive Zytoprotektion erreicht. In höheren, pharmakologisch wirksamen Dosierungen etwa ab $1000 \mu \mathrm{g}$ kann Selen durch verschiedene Selenmetabolite auch direkt zytotoxisch wirken. Dies ist ein Effekt, der synergistisch zur Zytotoxizität der Chemotherapie beitragen würde.

Stoffwechsel / Metabolisierung:

- Einbau über Selenocystein in Selenoproteine

- Ausscheidung über die Lunge als Methylselenol / Dimethylselenid und als Trimethyl-Selenium-Ion über die Nieren

Unerwünschte Arzneimittelinteraktionen:

Keine; eher Synergien mit zytotoxischer Chemotherapie/Strahlentherapie über zytotoxische Selenmetabolite wie Selenodiglutathion. Diese Selenmetabolite können einen zusätzlichen zytotoxischen Effekt und damit eine Wirkungsverstärkung mit sich bringen.

\section{Vitamin D}

Zufuhr in Form von Cholecalciferol

Indikationen in der Komplementärmedizin:

Supplementierung bei Mangel

Stoffwechsel, Metabolisierung:

- körpereigene Synthese: Umwandlung von Cholesterin zu 7-Dehydro-Cholesterin. Durch UV-B-Strahlung bei 290-315 nm entsteht in der Haut das Prävitamin D (Cholecalciferol). Es wird in die Leber transportiert, wo es zur Hydroxylierung in Calcidiol $(\mathrm{D}(25 \mathrm{OH}))$ kommt. In der Niere erfolgt die Umwandlung in das aktive Vitamin-D-Hormon (1,25-(OH)-D oder Calcitriol).

- Abbau: Mittels 24-Hydroxalase zu calcitriolischer Säure, dann Ausscheidung über die Niere in Form von Prävitamin D. Bei zu starker Sonneneinstrahlung kommt es zu Umwandlung in Lumisterol und Tachysterol.
Unerwünschte Arzneimittelinteraktionen:

Vitamin-D-Mangel kann durch Interaktion mit zahlreichen Medikamenten (Taxane, Tamoxifen, Aromatasehemmer) über den Pregnan-X-Rezeptor entstehen.

\section{L-Carnitin}

Indikationen in der Komplementärmedizin:

- Supplementierung bei Mangel

- interventionell als Prophylaxe und Therapie bei Fatigue-Syndrom

- Kardioprotektion unter Therapie mit Anthrazyklinen

- Neuroprotektion unter neurotoxischer Therapie (Taxane, Platinsalze)

Stoffwechsel / Metabolisierung:

- körpereigene Synthese; dafür benötigt der Organismus Co-Faktoren wie Lysin, Methionin, Vitamin C, Vitamin B6, Niacin und Eisen

- unveränderte Ausscheidung über die Niere, dort kommt es zu 90\%iger Rückresorption

Unerwünschte Arzneimittelinteraktionen:

- keine bekannten

- von L-Carnitin ausgehende Interaktionen mit medikamentöser Tumortherapie; L-Carnitin-Mangel durch pharmakokinetische Interaktion mit Ifosfamid, Cisplatin und Doxorubicin

\section{Coenzym Q10/Ubichinon 10}

Indikationen in der Komplementärmedizin:

- Elektronen- / Protonen-Überträger in der Atmungskette

- Supplementierung bei Mangel

- Supplementierung unter Therapie mit Statinen

- Kardioprotektion unter Therapie mit Anthrazyklinen

\section{Stoffwechsel / Metabolisierung:}

- Die Biosynthese erfolgt ausgehend von der 4-Hydroxybenzoesäure in komplexen Schritten bis zum Ubichinon 10.

- Verbrauch bei erhöhter oxidativer Belastung
Unerwünschte Arzneimittelinteraktionen:

- keine negative/ unerwünschte Interaktion mit Anthrazyklinen, anderen Zytostatika oder Tyrosinkinase-Inhibitoren

- Interaktionen mit Annonengewächsen (Graviola, Stachelannone, Acetogenine). Annonengewächse wie bestimmte Teile des Graviolabaums werden sehr intensiv in der traditionellen Naturheilkunde in Südamerika und Südostasien eingesetzt. Sie wirken gegen Parasiten und sollen krebsheilende Effekte haben. Acetogenine sind dabei die wesentlichen Wirkstoffe. Sie hemmen die Atmungskette/Komplex 1 in den Mitochondrien und könnten so zu unerwünschten Interaktionen mit Coenzym Q10/Ubichinon 10 führen [6].

\section{Vitamin C}

a) Enterale Zufuhr (bis 2g)

\section{Indikationen in der Komplementär-} medizin:

- Substitution von Mängeln (selten)

- Mangel durch Medikamenteninteraktionen

- Immunstimulation

- Co-Faktor im Eisenstoffwechsel

- Unterstützung der Wundheilung

Stoffwechsel / Metabolisierung:

- unveränderte Ausscheidung als Ascorbat über die Niere

- Der Abbau erfolgt wie bei Kohlenhydraten in $4 \mathrm{H}_{2} \mathrm{O}$ und $6 \mathrm{CO}_{2}$.

Achtung: Bei Nierensteinen, speziell bei Calcium-Oxalat-Steinen, vorsichtig mit der Zufuhr von Ascorbinsäure umgehen, da es eventuell zu einer Alkalisierung des Urins kommen kann.

\section{Unerwünschte Arzneimittelinteraktio-} nen:

Es kann zu Inhibition des Zytostatikums Proteasom-Inhibitor Bortezomib (Velcade) durch direkte Interaktion mit Bortezomib kommen.

b) Parenterale Zufuhr (15-100g i.v.) 


\section{Indikationen in der Komplementär-} medizin:

- Angestrebt bei der parenteralen Hochdosistherapie ist die selektive zytotoxische Wirkung auf Tumorzellen durch Peroxidbildung $\left(\mathrm{H}_{2} \mathrm{O}_{2}-\right.$ Peroxide)

- Ziel: Plasmalevel von>350 mg/dl (>20mM).

- In klinischen Studien mit Gemcitabin bei Pankreaskarzinom konnte kein negativer Einfluss auf die Chemotherapie gezeigt werden $[12,17]$.

\section{Stoffwechsel/ Metabolisierung:}

- unveränderte Ausscheidung als Ascorbat über die Niere

- Der Abbau erfolgt wie bei Kohlenhydraten in $4 \mathrm{H}_{2} \mathrm{O}$ und $6 \mathrm{CO}_{2}$.

Merke: Vor der Einleitung einer Hochdosis-Vitamin-C-Infusionstherapie ist unbedingt eine Bestimmung der Glucose-6-Phosphat-Dehydrogenase-Aktivität angezeigt. Bei Defekten oder verminderter Aktivität dieses En- zyms, beispielsweise bei Mutationsträgern, droht bei hochdosierter Vitamin-C-Infusion ( $a$ b 7,5g) eine Hämolyse.

Unerwünschte Arzneimittelinteraktionen:

Auch im Hochdosisbereich kann es zu Inhibition des Zytostatikums ProteasomInhibitor Bortezomib durch direkte Interaktion mit Bortezomib kommen.

\section{Bilanzierte Mikronährstoff- mischungen}

Indikationen in der Komplementärmedizin:

- Supplementierung bei Mangel

- Mangelernährung/Kachexie

- erhöhter Bedarf

Stoffwechsel/Metabolisierung:

Verläuft je nach Mikronährstoff unterschiedlich

Unerwünschte Arzneimittelinteraktionen:

Wenn Vitamin C enthalten ist: Achtung: Inhibition des Zytostatikums Proteasom-
Inhibitor Bortezomib durch direkte Interaktion mit Bortezomib

Aroniabeere / Aroniasaft Indikationen in der Komplementärmedizin:

- Immunstimulation

- antioxidative und antiinflammatorische Effekte

Stoffwechsel / Metabolisierung: Metabolisierung über CYP3A4 (Enzyminduktor); Chemotherapeutika wie z.B. Trabectedin werden ebenfalls über CYP3A4 metabolisiert. Bei Inhibition von CYP3A4 durch Aronia wird Trabectedin nicht abgebaut. Es kam so zu einer publizierten schwersten Rhabdomyolyse [15].

\section{Unerwünschte Arzneimittelinteraktio-} nen:

Enzyminhibition von CYP3A4, das für den Stoffwechsel beider Substanzen notwendig ist (Induktion / Stimulation). Somit kommt es zu Wirkungsverlust (Inhibition /Hemmung) und in der Folge zu Wirkungsverstärkung.

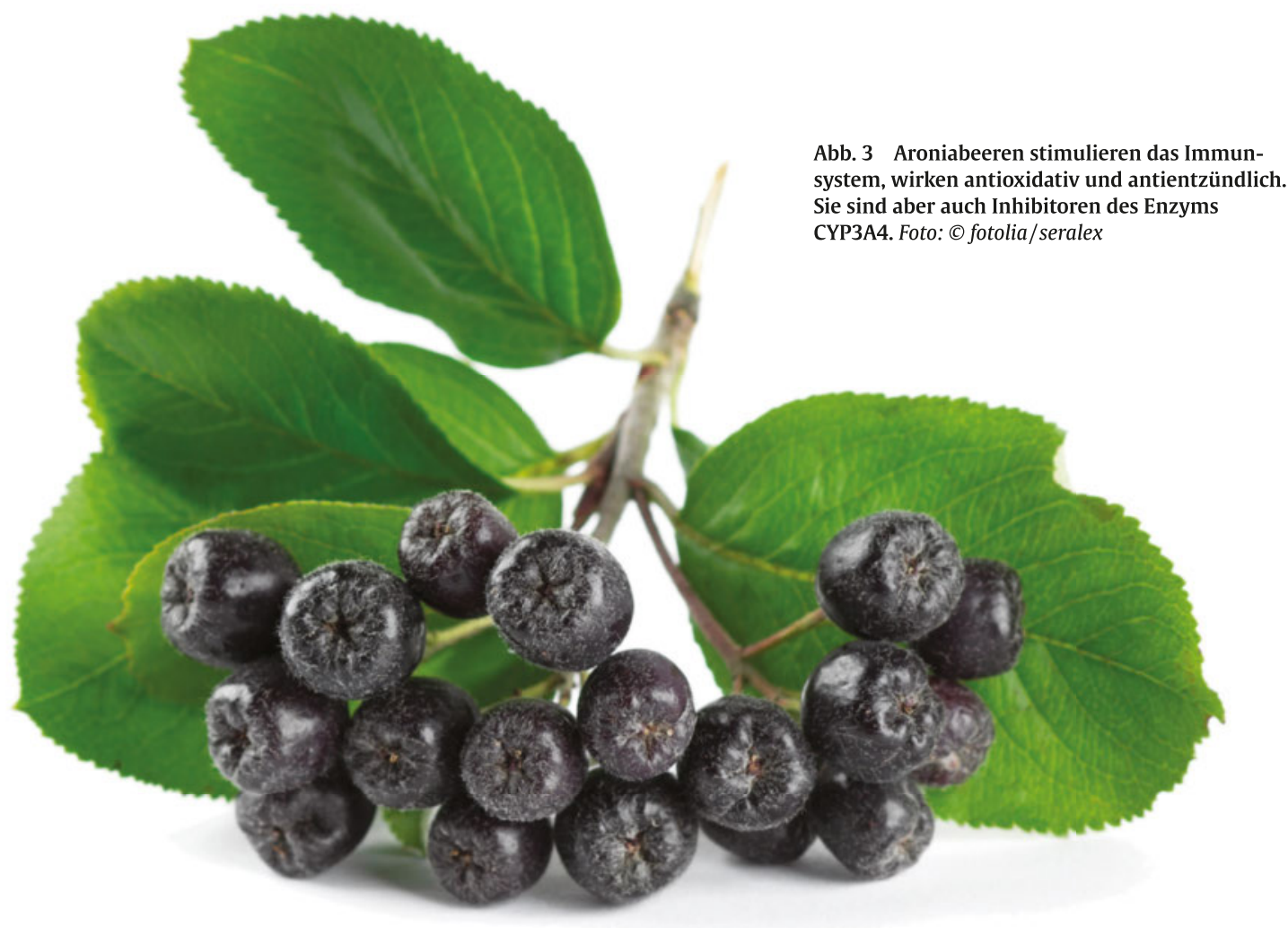




\section{Curcumin \\ Indikationen in der Komplementär- medizin: \\ - chemopräventive Effekte \\ - antiinflammatorische Effekte durch Inhibition von NF-kappaB, Cyclooxi- genase II, Lipoxigenase}

Stoffwechsel/ Metabolisierung:

- Synergie mit Vincristin, 5-FU und Paclitaxel

- antagonistische Wirkung zu grünem Tee

Erwünschte und unerwünschte Arzneimittelinteraktionen:

- Induktion der Glutathiontransferase und dadurch Verbesserung der Entgiftungsleistung in der Leber, Verstoffwechslung von krebsauslösenden Substanzen / Karzinogenen

- Antagonist des protektiv wirkenden EGCG (Epigallocatechin-3-Gallat) in grünem Tee

\section{Artemisin / Beifuß}

Indikationen in der Komplementärmedizin:

- zytotoxische Anwendung

- antiangiogenetische, proapoptotische und prooxidative Effekte

Stoffwechsel/ Metabolisierung:

Artemisine sind Induktoren von CYP2C19 und CYP3A4.

Unerwünschte Arzneimittelinteraktionen:

Artemisine können durch das Potenzial zur Induktion von CYP2C19 und CYP3A4 die Wirksamkeit von zahlreichen Arzneimitteln beeinflussen, die auch über das Cytochrom-P-450-System verstoffwechselt werden [14].

\section{Quercetin}

Indikationen in der Komplementärmedizin:

- Flavonoid mit antioxidativen und antiinflammatorischen, aber auch prooxidativen Eigenschaften

- Vorkommen in Äpfeln, Weintrauben, Zwiebeln, Brokkoli etc.
Stoffwechsel / Metabolisierung:

- Flavonoide modulieren die Cytochrom-P450-vermittelten Monooxygenaseaktivitäten.

- Quercetin, das am besten untersuchte Flavonoid, zeigt zusätzlich ein interessantes Wirkspektrum [3]. Es wirkt antientzündlich durch die Modulation der Eicosanoidbiosynthese, antiateriosklerotisch durch den Schutz der LDL-Fraktion vor Oxidation und antithrombotisch durch Hemmung der Plättchenaggregation.

Synergien/ unerwünschte Arzneimittelinteraktionen:

Synergie mit Cisplatin, Busulfan und Topotecan; Hemmung der Topoisomerase I und Hemmung von P-Glykoprotein. Dieses Protein fungiert als Entgiftungsprotein und schleust potenziell schädliche Fremdstoffe aus der Zelle. In Tumorzellen ist das Transportprotein ebenso nachgewiesen und es ist an der Elimination von verschiedenen Zytostatika beteiligt. Eine Hemmung durch Quercetin könnte möglicherweise einer Multi-Drug-Resistance, die in vielen Tumorzellen existiert, vorbeugen.

\section{Heilpilze}

Indikationen in der Komplementärmedizin:

- erwünschte Immunstimulation durch die verschiedenen Polysaccharide der Heilpilze: auf NK-Zellen, dendritische Zellen, Makrophagen, T-Lymphozyten und Stammzellen des Knochenmarks

- Bedeutung in der TCM und japanischen Medizin

Stoffwechsel / Metabolisierung:

Nicht bekannt

Merke: Wenn die Heilpilze aus Asien stammen, sind Schwermetallbelastungen möglich.

Unerwünschte Arzneimittelinteraktionen:

- Es sind keine negativen/unerwünschten Interaktionen mit zahlreichen untersuchten Zytostatika bekannt.
- Vermutet werden eher synergistische Wirkungen, auch mit der Strahlentherapie. Es liegen jedoch nur ältere Studien ohne modernes Studiendesign vor.

\section{Fazit}

Wie sich aus der vorausgegangenen Aufstellung ergibt, sind unerwünschte Wechselwirkungen durch Mikronährstoffe in der Schul- und Komplementärmedizin von geringerer Bedeutung $[1,2$, 6]. Die Metabolisierung ist hier meistens genau bekannt und gut geklärt. Problematischer ist das vielfältige Wechselwirkungspotenzial, das sich durch die Einbeziehung des Cytochrom-P450-Enzymsystems ergeben kann. Das betrifft zahlreiche Phytotherapeutika sowie eine fast unüberschaubare Anzahl von klassischen Medikamenten. Bei der heute, vor allem bei älteren Menschen üblichen Polypharmazie können diese Arzneimittelinteraktion schwerwiegende Folgen haben. Daher sollte jeder, der Medikamente rezeptiert, empfiehlt oder anwendet, auf die Kompatibilität der Einzelsubstanzen achten. Das sollte immer im Rahmen einer sorgfältigen Anamnese erfolgen. Hierbei ist es wichtig, auch nach scheinbar harmlosen Nahrungsmitteln zu fragen, die manchmal eben nicht für jeden und in jeder Kombination geeignet sind.

Dieser Artikel ist online zu finden: http://dx.doi.org/10.1055/s-0043-113060

\section{Tipps für den Alltag}

- sorgfältige Medikamentenanamnese

- Berücksichtigung von Naturstoffen, Vitalstoffen, Nahrungsmitteln

- bei Unsicherheiten bezüglich des Wechselwirkungspotenzials spezielle Datenbanken verwenden:

DRUG DIGEST (Express Script Inc (ESI), St. Louis MO, USA)

A.D.A.M. Drugchecker (Maryland MD, USA)

ABDA-Interaktionen (DIMDI Köln)

Der Arzneimittelbrief (Artikelsuche „Wechselwirkungen“) 


\section{Literatur}

[1] Alsanad SM, Howard RL, Williamson EM. An assessment of the impact of herb-drug combinations used by cancer patients. BMC Complement Altern Med 2016; 16(1): 393

[2] Alsanad SM, Williamson EM, Howard RL. Cancer patients at risk of herb / food supplement-drug interactions: a systematic review. Phytother Res 2014; 28(12): 1749-1755

[3] Andersen MR, Sweet E, Lowe KA et al. Dangerous combinations: Ingestible CAM supplement use during chemotherapy in patients with ovarian cancer. J Altern Complement Med 2013; 19(8): 714-720

[4] Block KI, Koch AC, Mead MN et al. Impact of antioxidant supplementation on chemotherapeutic toxicity: a systematic review of the evidence from randomized trials. Int J Cancer 2008; 123(6): 1227-1239

[5] Böhm R, Haen E. Arzneimittelinteraktionen. Verstehen, vermitteln und vermeiden. Deutsche Apothekerzeitung 2012; 36: 64

[6] Conklin KA. Cancer chemotherapy and antioxidants. J Nutr 2004; 134(11):3201-3204

[7] Espey MG, Chen P, Chalmers B et al. Pharmacologic ascorbate synergizes with gemcitabine in preclinical models of pancreatic cancer. Free Radic Biol Med 2011; 50(11): 1610 1619

[8] Ferri N, Bellosta S, Baldessin L et al. Pharmacokinetics interactions of monoclonal antibodies. Pharmacol Res 2016; 111: 592-599

[9] Hoffer L], Levine M, Assouline S et al. Phase I clinical trial of i.v. ascorbic acid in advanced malignancy. Ann Oncol 2008; 19(11): 19691974

[10] Loquai C, Dechent D, Garzarolli M et al. Risk of interactions between complementary and alternative medicine and medication for comorbidities in patients with melanoma. Med Oncol 2016; 33(5): 52

[11] Loya AM, González-Stuart A, Rivera JO. Prevalence of polypharmacy, polyherbacy, nutritional supplement use and potential product interactions among older adults living on the United States-Mexico border: a descriptive, questionnaire-based study. Drugs Aging 2009; 26(5): 423-436

[12] Ma Y, Chapman J, Levine M et al. High-dose parenteral ascorbate enhanced chemosensitivity of ovarian cancer and reduced toxicity of chemotherapy. Sci Transl Med 2014; 6(222): 222ra18

[13] Masuda Y, Inoue M, Miyata A et al. Maitake beta-glucan enhances therapeutic effect and reduces myelosupression and nephrotoxicity of cisplatin in mice. Int Immunopharmacol 2009; 9(5): 620-626
[14] Mooiman KD, Goey AK, Huijbregts T] et al. The in-vitro effect of complementary and alternative medicines on cytochrome P450 2C9 activity. J Pharm Pharmacol 2014; 66(9): 1339-1346

[15] Strippoli S, Lorusso V, Albano A. Herbal-drug interaction induced rhabdomyolysis in a liposarcoma patient receiving trabectedin. BMC Complement Altern Med 2013; 13: 199

[16] Sweet ES Standish LJ, Goff BA. Adverse events associated with complementary and alternative medicine use in ovarian cancer patients. Integr Cancer Ther 2013; 12(6): 508-516

[17] Welsh JL, Wagner BA, van't Erve T] et al. Pharmacological ascorbate with gemcitabine for the control of metastatic and node-positive pancreatic cancer (PACMAN): results from a phase I clinical trial. Cancer Chemother Pharmacol 2013; 71(3): 765-775

[18] Yang J, Bogni A, Schuetz EG et al. Etoposide pathway. Pharmacogenet Genomics 2009; 19(7): 552-553

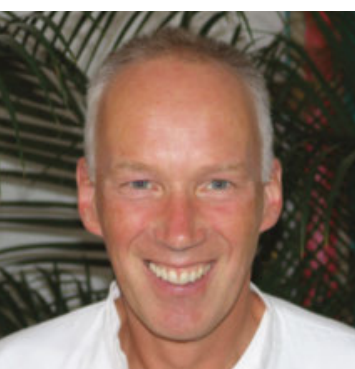

\section{Dr. med. Peter Holzhauer}

E-Mail: peter.holzhauer@klinik-bad-trissl.de oder peter.holzhauer@medicum-rosenheim.de

Dr. Peter Holzhauer ist Internist mit Zusatzbezeichnung Naturheilverfahren. Seit 2011 ist er Chefarzt der Abteilung Innere Medizin II / Onkologie und Komplementärmedizin der Klinik Bad Trissl in Oberaudorf. In der Privatpraxis IOZ München und in der Privatpraxis Medicum Rosenheim, Praxis für Integrative Medizin, bietet er jeweils eine onkologisch-komplementäre Sprechstunde an.

Zusätzlich ist er Mitherausgeber der Thieme-Fachzeitschrift „Deutsche Zeitschrift für Onkologie“ sowie Autor und Koautor verschiedener Fachbücher zum Thema onkologische Komplementärmedizin und Referent zahlreicher Workshops und Fachvorträge. 